\title{
A Signet Ring Cell Carcinoma Presented as Refractory Acquired Thrombotic Thrombocytopenic Purpura
}

\author{
Umit Yavuz Malkan $^{a} \quad$ Murat Albayrak $^{a} \quad$ Hacer Berna Ozturk ${ }^{a}$ \\ Merih Reis Aras ${ }^{a}$ Bugra Saglam ${ }^{a}$ Kutsal Dogan ${ }^{b}$ \\ aUniversity of Health Sciences, Diskapi Yildirim Beyazit Training and Research Hospital, \\ Department of Hematology, Ankara, Turkey; bUniversity of Health Sciences, Diskapi Yildirim \\ Beyazit Training and Research Hospital, Department of Pathology, Ankara, Turkey
}

\section{Keywords}

Microangiopathic hemolytic anemia - Thrombotic thrombocytopenic purpura - Signet ring cell carcinoma

\begin{abstract}
Microangiopathic hemolytic anemia (MAHA) can be observed as a paraneoplastic syndrome (PS) in certain tumors. MAHA-related signet ring cell carcinoma (SRCC) of an unknown origin is very infrequent. Herein we present a SRCC case presented with refractory acquired thrombotic thrombocytopenic purpura (TTP). A 35-year-old man applied to the emergency service with fatigue and headache. His laboratory tests resulted as white blood cell 9,020/ $\mu \mathrm{L}$, hemoglobin $3.5 \mathrm{~g} / \mathrm{dL}$, platelet 18,000/ $\mathrm{LL}$. Schistocytes, micro-spherocytes, and thrombocytopenia were observed in his blood smear. MAHA was present and he was considered as having TTP. Plasma exchange treatment was initiated; however, he was refractory to this treatment. Thorax and abdomen computerized tomography revealed thickening of minor curvature in stomach corpus with hepatogastric and paraceliac lymphadenopathy. Bone marrow (BM) investigation by our clinic resulted as the metastasis of adenocarcinoma. Ulceration and necrosis were observed by gastric endoscopy procedure. Biopsy was taken during endoscopic intervention, which resulted as SRCC. MAHA may be seen as a PS in some tumors, especially gastric cancers. Tumor-related MAHA is generally accompanied by BM metastases. As a result, BM investigation may be used as the main diagnostic method to find the underlying cancer. The clinical course of cases with tumor-related MAHA is usually poor, and these cases are usually refractory to plasma exchange treatment. In conclusion, physicians should suspect a malignancy and BM involvement when faced with a case of refractory TTP.


Malkan et al.: Signet Ring Cell Carcinoma with Thrombotic Thrombocytopenic Purpura

\section{Introduction}

Acquired thrombotic thrombocytopenic purpura (TTP) frequently presents as severe microangiopathic hemolytic anemia (MAHA) and thrombocytopenia in a previously healthy individual. Fatigue, dyspnea, petechiae, or other hemorrhagic events are usually the initial symptoms of MAHA and thrombocytopenia [1]. MAHA can appear as a paraneoplastic syndrome in certain tumors. MAHA is a late and mortal complication of metastatic cancer [2]. The most common tumors related with MAHA are gastric, breast, and lung cancers and malignancies of unknown origin $[3,4]$. However, MAHA-related signet ring cell carcinoma of unknown origin is very infrequent $[5,6]$. Herein we present a signet ring cell carcinoma case presented with refractory acquired TTP.

\section{Case Report}

A 35-year-old man applied to the emergency service with fatigue and headache on January 2020. In his anamnesis, he had a history of alcoholic pancreatitis. His physical examination was normal except for the neurological symptoms, which were temporary loss of consciousness and disorientation. His laboratory tests resulted as white blood cell $9,020 / \mu \mathrm{L}$, hemoglobin $3.5 \mathrm{~g} / \mathrm{dL}$, platelet 18,000/ $\mu \mathrm{L}$, MCV $110.7 \mathrm{fL}$, urea $58 \mathrm{mg} / \mathrm{dL}$, creatinine $0.84 \mathrm{mg} /$ dL, AST $68 \mathrm{U} / \mathrm{L}$, ALT $33 \mathrm{U} / \mathrm{L}$, indirect bilirubin $1.88 \mathrm{mg} / \mathrm{dL}$, LDH 2,257 U/L, reticulocyte $\% 10.1$, haptoglobulin $<8 \mathrm{mg} / \mathrm{dL}$, INR 1.42, prothrombin time 13.2, fibrinogen $184 \mathrm{mg} / \mathrm{dL}$, Coombs negative. He had consulted our clinic with bicytopenia and hemolysis. Schistocytes, micro-spherocytes, and thrombocytopenia were observed in his blood smear (Fig. 1). Microangiopathic hemolytic anemia was present and he was considered as having TTP. Plasma exchange treatment was initiated; however, he was refractory to this treatment. He had epistaxis and blurred vision during the follow-up. Superficial hemorrhages on the edges of the optic disc and Roth spots were detected. Pain had emerged in his right arm. Doppler ultrasonography revealed the occlusion of cephalic vein with non-recanalized thrombus in the subacute process from the antecubital level at the forearm level. Thorax and abdomen computerized tomography (CT) resulted as liver $220 \mathrm{~cm}$, spleen $14 \mathrm{~cm}$, minimal pleural effusion, thickening of minor curvature in stomach corpus with hepatogastric and paraceliac lymphadenopathy. As a result of $\mathrm{CT}$, endoscopic examination was planned. Bone marrow

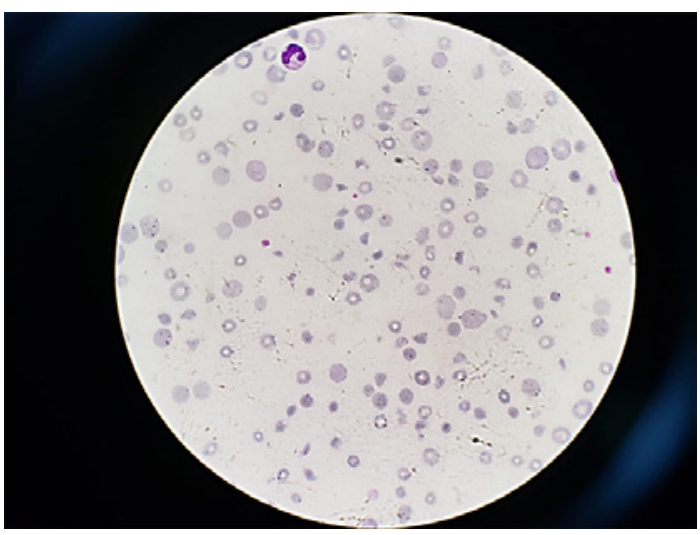

Fig. 1. Schistocytes, micro-spherocytes and thrombocytopenia were observed in the blood smear.

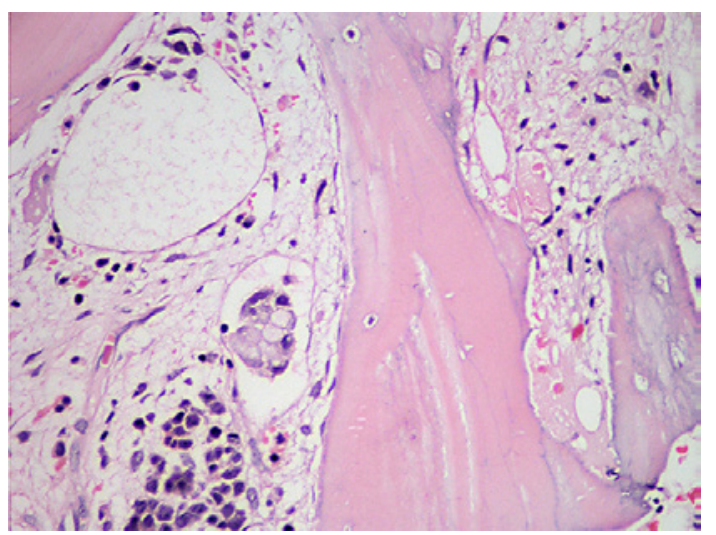

Fig. 2. Bone marrow biopsy under $\times 40$ magnification showing signet ring cells next to bone marrow trabecular $(\mathrm{H} \& \mathrm{E} \times 400)$. 


\section{Case Reports in Oncology}

Case Rep Oncol 2020;13:1368-1372 DOI: $10.1159 / 000510745$

(c) 2020 The Author(s). Published by S. Karger AG, Basel www.karger.com/cro

Malkan et al.: Signet Ring Cell Carcinoma with Thrombotic Thrombocytopenic Purpura

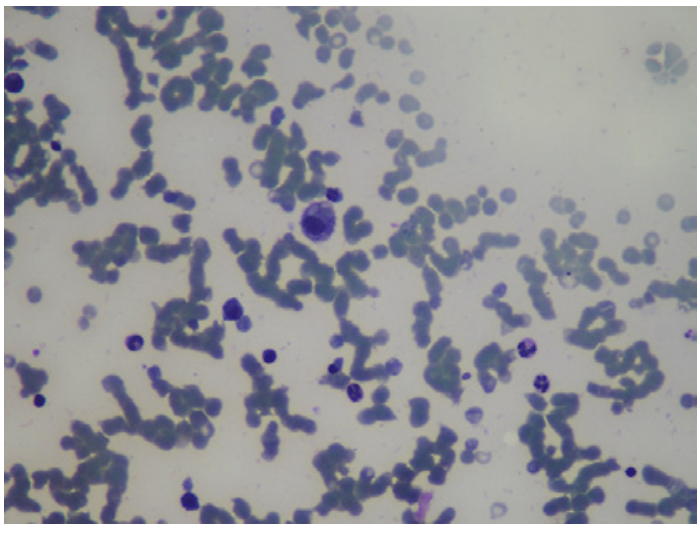

Fig. 3. Bone marrow aspiration under $\times 40$ showing a signet cell $(\mathrm{MGG} \times 400)$.

Fig. 5. Alcian blue-positive intracytoplasmic mucin of the signet cells for periodic acid Schiff-alcian blue (PAS-AB) staining $(\times 200)$.

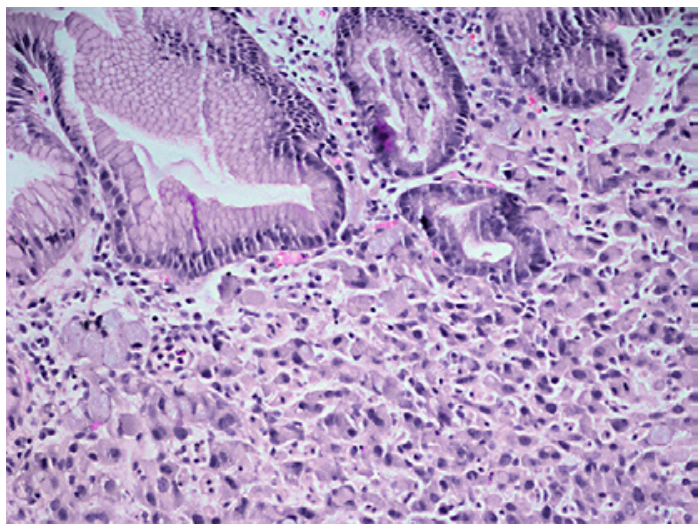

Fig. 4. Gastric endoscopic biopsy under $\times 20$ magnification showing signet cells beneath the normal gastric glands.

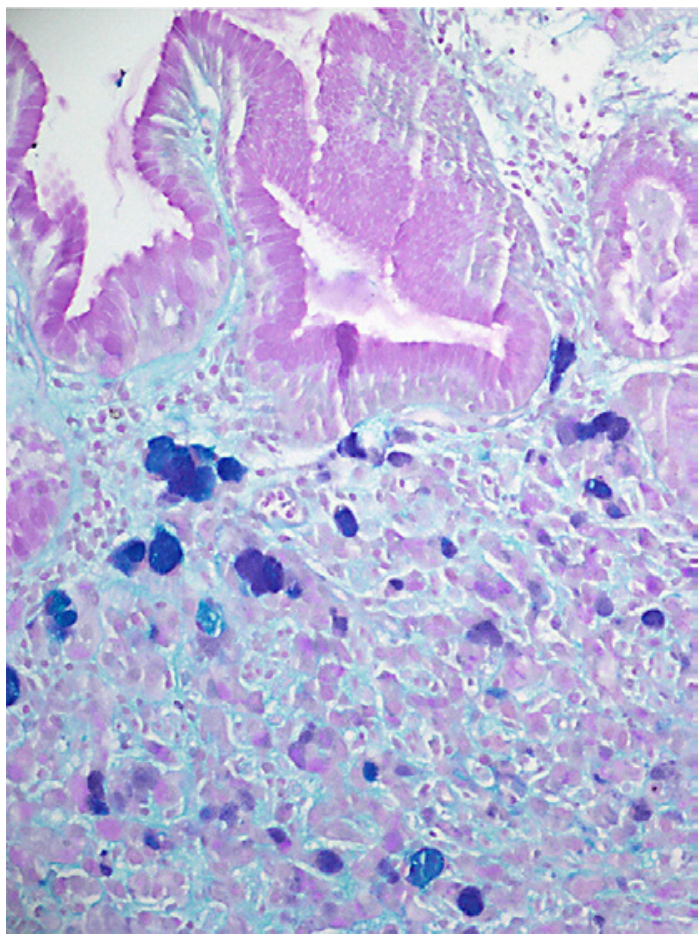

investigation by our clinic resulted as the metastasis of adenocarcinoma (Fig. 2, 3). Ulceration and necrosis were observed by gastric endoscopy procedure. Biopsy was taken during endoscopic intervention, which resulted as signet ring cell carcinoma (Fig. 4, 5). He was transferred to oncology clinic for his treatment. Unfortunately, he died in 1 month after his transfer.

\section{Discussion}

It is an important aspect in the clinical practice that not all patients with TTP are critically ill. TTP patients may present with minor complaints of weakness and dizziness, abdominal pain, easy bruising, or nausea and vomiting. Moreover, in some patients, the diagnosis of TTP 
may not be made until the laboratory test reveals severe thrombocytopenia and MAHA. Only $40 \%$ of TTP cases have the complete pentad and in $75 \%$ of the cases there is a triad of microangiopathic hemolytic anemia, thrombocytopenia, and neurological findings [7]. At present, the diagnosis of TTP is considered with microangiopathic hemolytic anemia and thrombocytopenia when any other cause is excluded [7]. In our case there was no acute kidney failure; however, all the other features favored TTP, and the diagnosis was made without kidney failure. MAHA can be observed as a paraneoplastic syndrome in some malignant conditions, particularly gastric tumors. The mechanisms of tumor-related MAHA include tumor-derived factors, procoagulants, immune complexes, some chemotherapeutic agents, fibrinoid necrosis of bone marrow, and tumor cell emboli of arteries, arterioles, and capillary [8]. Cancer-related MAHA is generally accompanied by multiple bone or bone marrow metastases [3]. Therefore, bone marrow investigation is useful as a primary diagnostic tool to examine the underlying cancer. Similarly, in our case, bone marrow investigation revealed the metastatic adenocarcinoma. Total plasma exchange is usually performed in the treatment of cancer-associated TTP; however, less than $20 \%$ of the cases respond to plasma exchange. Likely, our case did not respond to plasma exchange treatment either. The clinical course of cancer-associated TTP is generally poor. Patients are generally lost within 2 months due to multiorgan failure. Similarly, our patient had died soon after the diagnosis.

To summarize, MAHA may be seen as a paraneoplastic syndrome in some tumors, especially gastric cancer. Tumor-related MAHA is generally accompanied by bone marrow metastases. As a result, bone marrow investigation may be used as the main diagnostic method to find the underlying cancer. The clinical course of cases with tumor-related MAHA is usually poor, and these cases are usually refractory to plasma exchange treatment. In conclusion, physicians should suspect a malignancy and bone marrow involvement when faced with a case of refractory TTP.

\section{Statement of Ethics}

Written informed consent was obtained from the patient for the publication of the case report and any accompanying images.

\section{Conflict of Interest Statement}

The authors declared no potential conflicts of interest with respect to the research, authorship, and/or publication of this article.

\section{Funding Sources}

The authors received no financial support for the research, authorship, and/or publication of this article.

\section{Author Contributions}

All authors were involved in the preparation of this article. All authors have read and approved the final manuscript.

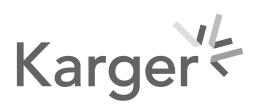




\section{References}

1 Griffin D, Al-Nouri ZL, Muthurajah D, Ross JR, Ballard RB, Terrell DR, et al. First symptoms in patients with thrombotic thrombocytopenic purpura: what are they and when do they occur?. Transfusion. 2013;53(1): 235-7.

2 Kwaan HC, Gordon LI. Thrombotic microangiopathy in the cancer patient. Acta Haematol. 2001;106(1-2): 52-6.

3 Shin SY, Park H, Chae SW, Woo HY. Microangiopathic hemolytic anemia as the first manifestation of metastatic signet ring cell carcinoma of unknown origin: a case report and review of literature. Korean J Lab Med. 2011; 31(3):157-61.

4 Niscola P, Palmieri MB, Scaramucci L, Vischini G, Giovannini M, Ferrannini M, et al. Fulminant thrombotic microangiopathy as a clinical presentation of an occult signet-ring cell carcinoma of the lung and misdiagnosed as idiopathic thrombotic thrombocytopenic purpura. Saudi Med J. 2010;31(5):581.

5 Eisa N, Nasef K, Emarah Z, Fattah MMA, Shamaa S. A Metastatic Signet Ring Cell Carcinoma Presented as Acquired Thrombotic Thrombocytopenic Purpura: A Case Report. J Hematol. 2018;7(2):72-5.

6 Otrock ZK, Taher AT, Makarem JA, Kattar MM, Nsouli G, Shamseddine AI. Thrombotic thrombocytopenic purpura and bone marrow necrosis associated with disseminated gastric cancer. Dig Dis Sci. 2007;52(6): 1589-91.

7 Kadikoylu G, Barutca S, Tataroglu C, Kafkas S, Erpek H, Meydan N, et al. Thrombotic thrombocytopenic purpura as the first manifestation of metastatic adenocarcinoma in a young woman. Transfus Apher Sci. 2010;42(1): $39-42$.

8 Yuce T, Bakkaloğlu OK, Köse M, Akpınar TS, Tükek T. Microangiopathic hemolytic anemia in metastasized signet ring cell carcinoma: a report of three cases. International Journal of Hematology Research. 2016;2: 136-8. 